\title{
Testing the Sustainability of Growth of the LJSEX in the January 2000 to May 2010 period
}

\begin{abstract}
Aleš Ahčan
Faculty of Economics, University of Ljubljana, Kardeljeva ploščad 17, SI-1000 Ljubljana, Slovenia, ales.ahcan@ef.uni-lj.si

In this paper we analyse the behaviour of the LJSEX, the main index of the Ljubljana Stock Exchange, in the period from January 2000 till May 2010. More precisely, we test for the presence of bubbles and antibubbles and try to determine whether or not a bubble could have been predicted (both the formation and the date of the bubble burst). Second, we also employ techniques used to model antibubbles to forecast the future behaviour of the LJSE index. Besides modelling index dynamics for the aforementioned period, we also seek to determine the factors that led to the bubble forming and later bursting. We find that the bubble could have been forecasted at least several months in advance. On the other hand, a very precise date of the crash seems harder to identify. By more closely analysing the interplay between interest rates, credit activity and the LJSEX, we conclude that there is a clear connection between decreasing interest rates, increased credit activity and the formation of a stock bubble. If there is a clear correlation between the early phase of a bubble and increased credit activity of the banking sector, the link between the end of the bubble and the restriction of credit activity is less pronounced. By fitting the extended antibubble model from (Johansen 1999a) we obtain the values of parameters that give us some indication of the future behaviour of the LJSEX. Based on these results we conclude that in the next few years we are likely to experience a period of increased volatility with no clear increasing or decreasing growth pattern.
\end{abstract}

Keywords: bubbles, antibubbles, LPPL, forecast

\section{Introduction}

It has been known for some time that markets can behave in a speculative fashion that may result in price bubbles, with prices reaching levels that are unjustified by economic fundamentals. One of the most prominent models used to detect stock bubbles was developed by Sornette and Johansen (see for example Johansen et al. $\left.\left(1999 \mathrm{a}, 1999_{b}\right)\right)$ where the authors used ideas developed in statistical physics (i.e.. the ISING model) to describe price dynamics. According to (Johansen et al. 1998,1999 a,b), prices can be described by the so-called log periodic power law (LPPL), whereby using several indicators one can both determine the resulting bubble and the time at which the transition to another phase will occur. There is a clear analogy between phase transitions and stock bubbles since in both cases agents (either investors or particles) exhibit similar behaviour/agree on the characteristics of the "surrounding media".

There is ample evidence that most of the stock market has experienced some sort of bubble-like behaviour. A short but far from complete list identifies a list of past bubbles that appeared in the USA (Dow Jones 1929, S\&P 1987), Japan (Nikkei 1989) the Hong-Kong Oct. 1997 crash and more recently the South Africa stock bubble in the 2003-2006 period, the recent UK and US real estate bubble, the 2009 Chinese equity bubble, and the CDS bubble in 2009.

As proven by Sornette and Johansen (1999, 2000), similar herding behaviour can be observed in the case of decelerating market devaluations following all-time highs. Such behaviour has been confirmed in 21 stock market indices: the Netherlands, France, the USA Dow Jones, USA NASDAQ, Japan, Belgium, Denmark, Germany, Norway, Spain, Switzerland, the United Kingdom, Israel, Brazil, Hong Kong, India, Peru, Taiwan, Czech Republic, Argentina and Turkey.

It also appears there is evidence that bubbles formed and were present in the economies of the former Soviet Union and in countries in transition. Yet no one has sought to test for the presence of bubbles in the case of Slovenia. Slovenia was one of the countries that followed a pre-determined exchange rate depreciation path ${ }^{1}$ (Capriolo et al., 2003). Accordingly, inter-

1 The monetary policy framework in Slovenia has been modified three times since country's independence in 1991. First, price stabilization was pursued with a framework that relied on monetary anchor (1991-1995). After a single digit inflation level was achieved, the stability of the 
est rates were relatively high in both nominal and real terms and started dropping only after Slovenia joined the euro zone. Thus, an analysis of the dynamics of the Slovenian stock market is interesting from several viewpoints. Two main questions need to be answered, namely, was a bubble present in Slovenia in the years of easy credit (especially in the period from 2002 to 2008) and, second, to what extent did the situation in the Slovenian banking sector influence the formation of a bubble?

In this paper we remedy this situation. We analyse the behaviour of the LJSEX, the main index of the Ljubljana Stock Exchange (LJSE), in the period from January 2000 till May 2010. More precisely, we test for the presence of bubbles and antibubbles and try to determine whether or not a bubble could have been predicted (both the formation and the date of the bubble burst). Second, we employ techniques used to model antibubbles to forecast the future behaviour of the LJSE index. Besides modelling the index's dynamics for the aforementioned period, we also try to determine the factors that led to a bubble being formed and later bursting.

We find conclusive evidence using both the simple test for non-linear exponential growth presented in Zhou and Sornette (2007) and the methods of statistical physics (LPPL model) developed in (Johansen et al. 1998,1999 a,b) for the presence of a stock bubble. Regarding the possibility of predicting the bursting of a bubble ex ante, we find evidence that the end of the bubble could have been predicted, although the exact timing of it bursting would be harder to predict. The evidence of an antibubble phase is less clear especially because the power exponent is a little high. If, however, one assumes that we are currently in an antibubble phase of the market we can expect a period of increased volatility with no apparent downward or upward trend in the next year. With respect to the interplay between interest rates and LJSEX levels, we find a strong correlation between a reduction of interest rates and growth of the LJSEX. Moreover, the growth of credit coincides well with the beginning of the bubble. The credit activity of banks picked up significantly in 2005, 2006 and 2007. Although there is a clear correlation between the early phase of the bubble and the increased credit activity of the banking sector, the link between the end of the bubble and the restriction of credit activity is less pronounced. With respect to the future behaviour of the LJSEX using the methodology of antibubbles introduced in (Johansen et al. 1999 ), the most likely outcome seems to be a pattern of oscillatory behaviour of the stock index with no apparent upward or downward trend.

In Section 2 we briefly overview the methodology used to analyse bubbles and antibubbles. In Section 3 we introduce our data set along with its main characteristics. Section 4 outlines the results of the analysis of the dynamics of the LJSE index. In this section we provide a simple analysis that confirms the presence of the bubble and then complement it with the more advanced approach of Sornette and Johansen (see Johansen et al. 1998,1999a,b). At the end of this section we also give a best estimate of the future dynamics of the
LJSEX using the Sornette and Johansen method for predicting antibubbles (Johansen et al. 1999a ). In Section 6 we try to determine the factors that influenced the forming and bursting of the bubble. Section 7 concludes.

\section{Research methods}

In this section we briefly overview the methodology used to detect bubbles and antibubbles. A more complete description of the methodology can be found in Johansen et al. $1998,1999_{a, b}, 2000_{a, b}$ ). For some criticism of the Johansen and Sornette approach, see Feigenbaum (2001 a,b). One should note that the methodology in this paper differs from the mainstream literature developed in 1980's and 1990's. Some of the main contributions of the early work on bubbles can be found in Diba et al (1988). Blanchard (1979), West (1987), Froot et al (1991).

\subsection{Modelling Bubbles}

As confirmed by numerous authors (see for example Johansen et al. 1998,1999 a,b), a bubble can be identified by the existence of a regime of prices (either stock market or real estate) well described by the expression

Here A represents a constant or value of a price index obtained at the peak of a bubble, the term $\left(t-t_{c}\right)^{m}$ captures faster than exponential growth of the price index (with $\mathrm{m}$ limited to $[0,1])$ and $\left(\cos \left(\omega\left(\log \left(t-t_{\downarrow} c\right)\right)-\phi\right)\right.$ describes the oscillation of the price index around an exponential path, with $t_{c}$ denoting the time of the crash or more generally of a phase transition, $\varphi$ is a phase shift, $C$ gives the size of oscillation amplitude and $\mathrm{B}$ (relative to $\mathrm{A}$ ) is the share of the value of the index that can be attributed to faster than exponential growth. As noted by Johansen and Sornette $(1999 \mathrm{a}), 3$ of the 7 parameters $(A, B, C)$ depend on the values of the other four parameters and thus the optimisation problem depends on the values of the remaining 4 parameters. Of the 7 parameters only $\mathrm{m}$ and $\omega$ carry structural information about the market dynamics. This is further emphasised by numerous studies of past bubbles confirming that the values of parameters $m$ and $\omega$ take on specific values (see Johansen and Sornette, Log-periodic power law bubbles in Latin-American and Asian markets and correlated anti-bubbles in Western stock markets: An empirical study). Testing the aforementioned methodology in the case of Latin American and Asian markets they find evidence that for the majority of bubbles the power exponent should take values in the range $0.1<\mathrm{m}<.8$ (Sornette et al. 2001), whereas the values for the angular frequency are in the range $2.9<\omega<11.4$.

currency measured both in terms of prices and the real exchange rate was pursued by means of dual targeting of both base money and the exchange rate (1996-2001). The last change in the monetary framework, aiming at addressing the persistence of inflation and the

EU accession requirements, rests on a framework that uses the exchange rate as a nominal anchor for reducing inflation (2001- 2004) 
In a series of papers (Johansen et al. 1998,1999a,b, Sornette et al. 2001), Johansen and Sornette demonstrate that most stock market bubbles can be identified using the methodology described above. In addition, they demonstrate that most of the bubbles that appeared could have been identified up to 2 years in advance and in some cases even 5 years in advance.

\subsection{Modelling Antibubbles.}

As pointed out by Sornette and Johansen (Johansen et al. 1999 a), a similar type of herding behaviour leading to $\log$ periodic oscillations observed during a bubble phase may also be observed during a post-bubble or crash phase.

Such behaviour has been identified in 21 stock market indices: the Netherlands, France, the USA Dow Jones, USA NASDAQ, Japan, Belgium, Denmark, Germany, Norway, Spain, Switzerland, the United Kingdom, Israel, Brazil, Hong Kong, India, Peru, Taiwan, Czech Republic, Argentina and Turkey. Thus in analogy with (1) where Sornette and Johansen propose that the price dynamics in the post-bubble phase are modelled as

$\operatorname{In}(p(t))=A+B\left(t_{c}-t\right)^{m}+\left(t_{c}-t\right)^{m}\left(\cos \left(\omega\left(\log \left(t_{c}-t\right)\right)-\phi\right)\right.$

With $t>t_{c}, t_{c}$ as before denoting the most likely time of phase transition from the bubble to antibubble phase, $\mathrm{C}$ denoting the size of the oscillation. Observe that equation 2 is basically a transformed equation 1 with $\mathrm{t}-\mathrm{t}_{\mathrm{c}}$ being replaced by $\mathrm{t}_{\mathrm{c}}$ - $\mathrm{t}$.

Besides the simple first order harmonics, in their article (Johansen 1999a) Sornette and Johansen also propose an extension to equation 2 by including second order harmonics

$$
\begin{aligned}
& \ln (p(t))=A+\frac{\tau^{m}}{\sqrt{1+\left(\frac{\tau}{\Delta t}\right)^{2 m}}}(B+C \cos \{\omega \log \tau+ \\
& \left.+\frac{\Delta \omega}{2 m}\left(1+\left(\frac{\tau}{\Delta t}\right)^{2 m}\right)+\phi\right\}
\end{aligned}
$$

The main difference between equations 2 and 3 is the inclusion of additional terms which allow for a change of oscillatory pattern from $\omega$ close to $t_{c}$ to $\omega+\Delta \omega$ far from $t_{c}$ (i.e. for $\mathrm{t}-\mathrm{t}_{\mathrm{c}}>\Delta \mathrm{t}$ ).

\section{Results}

\subsection{The dynamics of the LJSE index in the 2000-2010 period}

Although the LJSE was established in 1989 it was not until 1996 that the first paper was traded on the LJSE. It was only in mid-2000 that the first stock index was established. Today there are two main stock indexes: SBI TOP and LJSEX.

In this paper we focus on the LJSEX which comprises the biggest stocks traded on the LJSE: The dynamics of the LJSEX are depicted in Figure 1.

As one can see from the figure, for the period between 2000 and 2006 the stock market experienced a period of more or less positive returns, with intermediate periods of minor corrections. For the first five years the compounded return was equal to around $20 \%$, with a standard deviation of around $20 \%$. Although high in nominal terms, the return was somewhat smaller in real terms due to relatively high inflation. One should note that inflation in Slovenia was high at the begin-

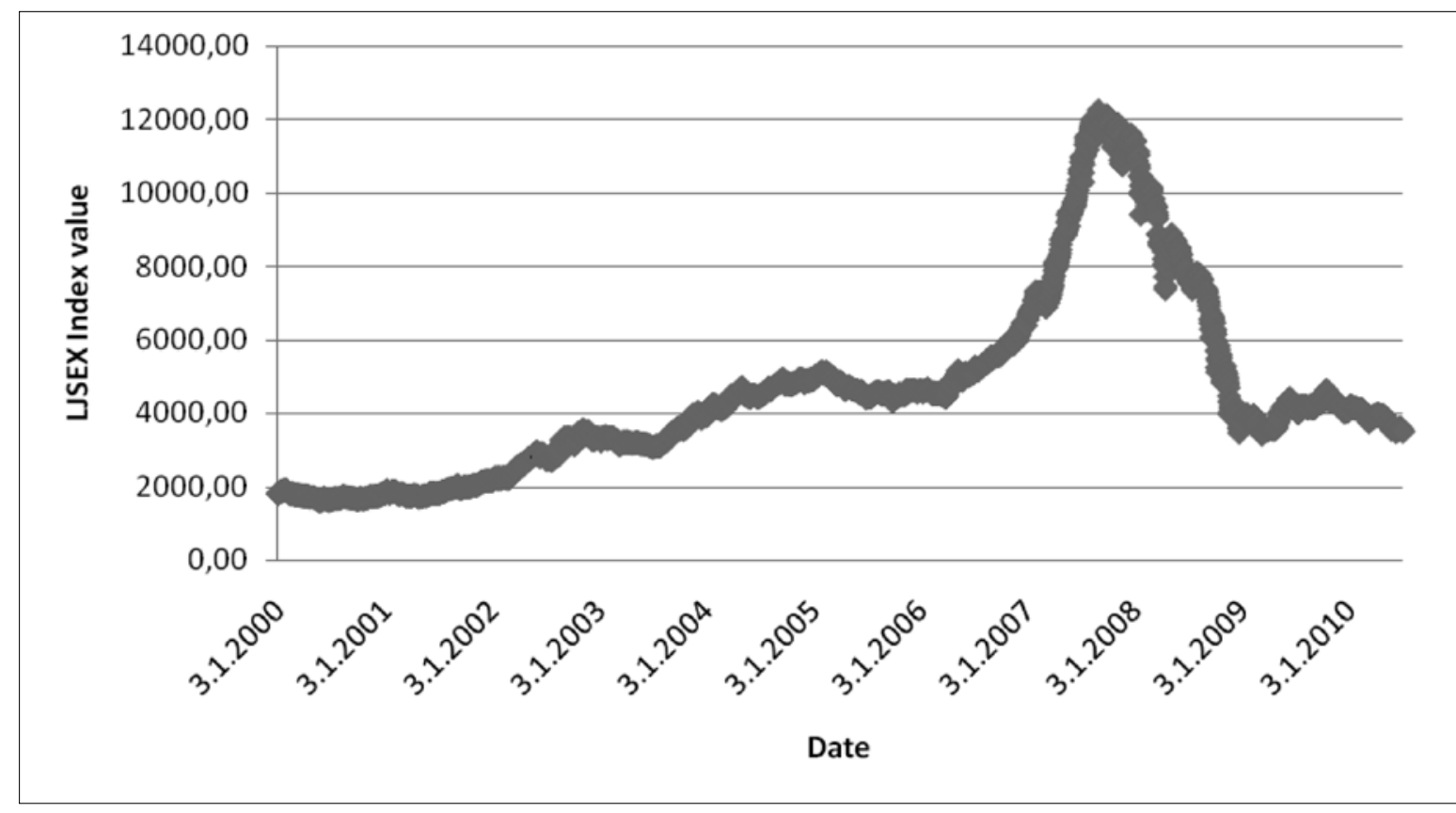

Figure 1:The dynamics of the LJSEX in the period January 2000 to May 2010. The x axis denotes date, while the y axis gives the value of the LJSEX. 
ning of the new millennium. Namely, in 2000 inflation was close to $9 \%$, it was similar in 2001, and then dropped gradually to around $4 \%$ in 2004 . Correspondingly, during the same time we also witnessed the continuous depreciation of the Slovenian tolar (SIT), the local currency of the time, and experienced a period of relatively high interest rates, with interest for short-term credit exceeding $10 \%$ at the beginning of 2000 .

A real change of regime (as it would appear) happened in the middle of 2006 when the index started growing rapidly. In the last half of 2006 the index returned to close to $30 \%$, whereas during the next 9 months the index returned a record $92 \%$. During this time the returns were almost uniformly positive and periods of constant growth were only occasionally interrupted by one or two days of minimal corrections. The index culminated on 31.8.2007 and started what seemed at first to be a slow descent from record heights. At the beginning of 2008 the index was still only $5 \%$ below its record levels. A real correction came in 2008 when the index lost more than $70 \%$ of its value. After that date, the index stabilised somewhat with small oscillations around the 4,000 points mark.

At first glance it would seem that in the period between 2006 to end of 2007 we experienced a classical stock bubble that ended in a swift crash. Although this hypothesis seems robust from the data we have presented, we still subject this hypothesis to further tests by more carefully analysing the stock market's behaviour in this period using the methodology of Sornete and Johansen (see for example Johansen et al. 1998,1999 a,b).

\subsection{Was there a bubble?}

In the first part of our analysis using a simple test we will try to determine whether the presence of the bubble could have been detected. For these purposes, we use the simple method proposed by (Zhou et al. 2007). Namely, the presence of a bubble can be identified if one compares the quality of the fit of the model describing super-exponential growth with the fit of the standard exponential growth model. Here the standard exponential growth model is characterised by the following equation

$\operatorname{In}(\mathrm{p}(\mathrm{t}))=\mathrm{A}+\mathrm{Bt}+\varepsilon_{1}$

Where $\varepsilon_{1}$ stands for the random walk component and $\mathrm{b}$ is the expected return (compounded) per unit of time (here 1 trading day is chosen as a suitable time unit). On the other hand, in the most simple form the super-exponential growth model is characterised by the following equation

$$
\operatorname{In}(\mathrm{p}(\mathrm{t}))=\mathrm{A}+\mathrm{Bt} \mathrm{Ct}^{2}+\varepsilon_{1}
$$

where the non-linear effect of the super-exponential growth is captured via the additional quadratic term. As mentioned, over a given time interval we determine whether or not a bubble existed by comparing the standard deviation of errors of a linear model (fitted over a given time interval) with the standard deviation of errors of the simple non-linear model with a quadratic term. If the standard deviation of the errors of the non-linear model is much smaller than the standard deviation of the errors of the linear model this might indicate that the dynamics of the return over a given time horizon were distinctively non-linear and could indicate the presence of a bubble. More precisely, Sornette and Zhou (Zhou et al. 2007) use the following metric $\mathrm{D}$ to determine the presence of a bubble

$$
\mathrm{D}=\frac{\mathrm{RMS}(\operatorname{lin})-\mathrm{RMS}(\text { non })}{\mathrm{RMS}(\operatorname{lin})}
$$

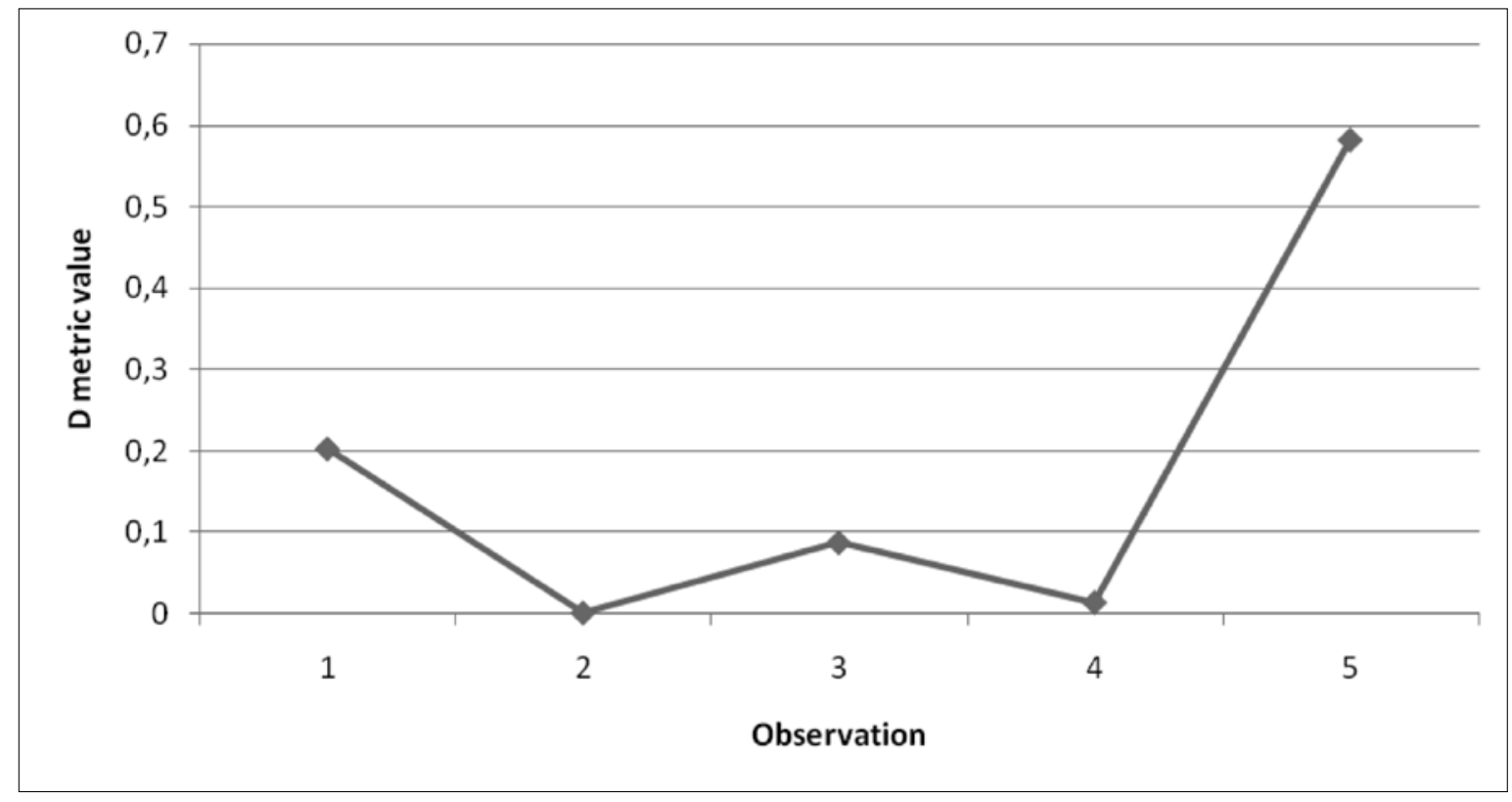

Figure 2:D metric indicating bubble-like behaviour for the period between 3 January 2000 and 31 August 2007. On $y$ axis the values of D metric are given and on x axis the time intervals (380 days long) starting on 3 January 2000 are given. Source: LJSE 
Where RMS(lin) is the root-mean-square of the residuals of the fits to the price time series with the linear model and RMS(nonlin) is the root-mean-square of the residuals of the fits to the price time series with the non-linear model. As determined by Zhou and Sornette (Zhou et al. 2007), values of index $\mathrm{D}$ higher than 0.25 indicate the presence of a bubble.

In order to test for the presence of a bubble in the Slovenian market we use data from 3 January 2000 until 31 August 2007 and use the aforementioned methodology to calculate the value of index D using both linear and nonlinear models. First, we test to what extent the values of the index differ over a series of non-overlapping (time) intervals obtained by splitting the time period from 3 January until 31 August 2007 into five equally spaced time intervals. There are approximately 1,900 trading days between 3 January 2000 and 31 August 2007, so each time interval is 380 trading days long.

As we can see from Figure 2 the values of index D are smaller than 0.25 for all time intervals except the last one. Thus it seems that distinct bubble behaviour was present only in the last time interval between 31 January 2006 and 16 August 2007. For all other time intervals the values of the index are so small that we may exclude the presence of a bubble. From the above analysis we may conclude that the results of the comparison between the simple linear and nonlinear models indicate it is very likely that a bubble existed in the period between 2006 and the summer of 2007. The test results also suggest that the bubble began sometime in the period between the beginning of the fourth time interval (29 July 2004) and the beginning of the last interval (31 January 2006).

One would of course like to more accurately determine the beginning of the bubble and also test whether or not the end of the bubble could have been forecast in advance. In order to do this, we focus our attention on an analysis of the LJSE dynamics between January 2000 and September 2007 using the more advanced methodology presented in Zhou et al. (2007).

\subsection{When did it all begin? Identifying the start of the bubble}

An important question here is whether one can determine the beginning of the bubble phase, how accurately and how far in advance did the signals identify the presence of the bubble. Following Sornette and Zhou (2007), we use a method of decreasing time windows where we fix the end of the time horizon (denoted $\left.\mathrm{t}_{\text {last }}\right)$ and vary the length of the time horizon by changing $\mathrm{t}_{\text {start }}$. On such a time horizon $\left(\mathrm{t}_{\text {start }}, \mathrm{t}_{\text {last }}\right)$ we search for a minimum by using least squares with a distance equal to the difference between the value of the index and the value of the approximating sequence given by equation (1). In order to identify a global minimum between many local minima we use a variant of a taboo search (Cvijovic et al. 1995). Having obtained estimates for the parameters in equation (1) that identify global minima, we change the size of the time interval and repeat the procedure of identifying global minima. Once the value of critical time $t_{c}$ does not change significantly (e.g. by more than a few months) when increasing $\mathrm{t}_{\text {start }}$ this can be identified as the beginning of the bubble.
In order to identify the start of the bubble we performed the aforementioned analysis on the whole data set, with $\mathrm{t}_{\text {last }}$ set at 10 August 2007, and change $t_{\text {start }}$, beginning on 3 January 2000 (the beginning of our data set). (Since we are concerned with identifying ex post when the bubble started we use as many data points as possible to make the estimates robust, we thus take the last point in the time set that is close to the actual peak of the bubble).

\section{Table 1: $T_{c}$ as a function of $t_{\text {start }}$ using the method of decreasing time windows using a variant of a Taboo search}

\begin{tabular}{|l|l|}
\hline$T_{\text {start }}$ & $\mathrm{T}_{\text {critical }}$ \\
\hline 06-Jan-2000 & 20-Aug-2008 \\
\hline 23-Oct-2000 & 21-Nov-2008 \\
\hline 11-Aug-2001 & 29-Apr-2008 \\
\hline 30-May-2002 & 05-Jun-2008 \\
\hline 18-Mar-2003 & 16-Dec-2010 \\
\hline 04-Jan-2004 & 02-May-2018 \\
\hline 22-Oct-2004 & 09-Nov-2011 \\
\hline 10-Aug-2005 & 31-Aug-2007 \\
\hline 08-Sep-2005 & 15-Sep-2007 \\
\hline 07-Oct-2005 & 28-Aug-2007 \\
\hline 05-Nov-2005 & 18-Sep-2007 \\
\hline 04-Dec-2005 & 01-Sep-2007 \\
\hline 03-Jan-2006 & 15-Sep-2007 \\
\hline 01-Feb-2006 & 29-Aug-2007 \\
\hline 02-Mar-2006 & 13-Sep-2007 \\
\hline
\end{tabular}

Source: LJSE and own work

As one can see from the table, the values of $t_{c}$ (critical time) vary quite significantly over the whole data set. Up to approximately August 2005 the estimates of critical time vary substantially ${ }^{2}$. As the table shows, after that time the estimates of critical time become very robust, the estimates of the time of the phase shift, i.e. critical time, are almost uniform (the dates vary only slightly with most of them focussing on the period from the end of August to mid-September). One should note that use of the methodology in this section yields similar results for the beginning of the bubble as with the analysis employing the simpler methods from the previous section.

\subsection{Could the end of the bubble have been predicted?}

Looking ex post we know that the bubble culminated on 31 August resulting in a large correction over the next three years. From the viewpoint of the methodology proposed by Johansen and Sornette (Johansen et al. 1998), we would like to know whether the end of the bubble could have been predicted and, if so, how far in advance? To test this hypothesis we adopt the method of increasing time windows proposed by Zhou et al. (2007) where we fix a start date $t_{\text {start }}$ which is identified as the most likely start of the bubble and change $t_{\text {last }}$ (indicating the end time point of the time window). Searching for the 
best fit and increasing $t_{\text {last }}$ gives us the dependence of $t_{c}$ as a function of $t_{\text {last }}$. When $t_{c}$ becomes fairly robust or does not change with respect to $t_{\text {last }}$, this can be interpreted as evidence of a possible regime shift (bursting of a bubble). For the purpose of our analysis we fix $t_{\text {start }}$ as August 2005 which was previously identified as the most likely start date of the stock bubble. For $t_{\text {last }}$ we consider months between October 2006 and September 2007.

Table 2: Values of the parameters obtained by changing $t_{\text {last }}$ using the method of increasing time windows

\begin{tabular}{|l|r|r|l|}
\hline $\mathrm{t}_{\text {start }}$ & \multicolumn{1}{l|}{$\mathrm{m}$} & \multicolumn{1}{l|}{$\mathrm{t}_{\mathrm{c}}$} \\
\hline 30.5 .2006 & 0.989122 & 1.608058 & 21.8 .2007 \\
\hline 27.6 .2006 & 0.016345 & 4.09723 & 17.7 .2006 \\
\hline 25.7 .2006 & 0.040763 & 4.669221 & 25.8 .2006 \\
\hline 18.10 .2006 & 0.212778 & 19.45637 & 18.10 .2007 \\
\hline 17.11 .2006 & 0.454071 & 19.988 & 10.1 .2008 \\
\hline 15.12 .2006 & 0.526271 & 5.153285 & 18.12 .2006 \\
\hline 22.1 .2007 & 0.161625 & 4.841301 & 16.2 .2007 \\
\hline 20.2 .2007 & 0.009689 & 5.993176 & 2.4 .2007 \\
\hline 20.3 .2007 & 0.357402 & 1.169174 & 11.4 .2007 \\
\hline 19.4 .2007 & 0.040652 & 2.446444 & 14.5 .2007 \\
\hline 22.5 .2007 & 0.005136 & 8.950006 & 12.7 .2007 \\
\hline 19.6 .2007 & 0.641984 & 0.404496 & 29.5 .2008 \\
\hline 18.7 .2007 & 0.389706 & 0.47664 & 21.8 .2008 \\
\hline 16.8 .2007 & 0.241565 & 0.837862 & 23.8 .2007 \\
\hline 13.9 .2007 & 0.493021 & 0.40956 & 23.8 .2007 \\
\hline
\end{tabular}

Source: data LJSE and own work

The table shows that in the case of the LJSE index after March 2007 the estimates of critical time become more robust. Namely, between February 2007 and September 2007 critical time varies between August 2008 and January 2007, with a relatively stable estimate of critical time between August 2007 and September 2007 for the last eight estimates. Thus, the answer to the question of whether the burst of the bubble could have been forecast is a clear yes, although the exact date of the phase regime change would seem harder to determine. Further, the results of the power exponent $m$ confirm the presence of the bubble; in only a few rare cases does the value of the exponent fall outside the optimal range $0.1<\mathrm{m}<.8$. On the other hand, the values of the angular frequency do not fall within the predetermined range that is common to most bubbles. Namely, the values of angular frequency are in most cases much smaller than is the case for other known bubbles. We believe that part of the explanation may be linked to the fact that the LJSE bubble evolved over a shorter time period than comparable bubbles studied by other authors (Johansen et al. 1998,1999 a,b). Hence the log periodicity became less apparent than it would have had the bubble formed over a longer time span.

Although the evidence regarding angular frequency is less convincing than, for example, the values of the power exponent or the values of the comparison between the linear and quadratic models, we believe there was ample evidence of a bubble forming well before the crash on 31 August 2007. Moreover, both analyses conducted in the previous two subsections support the reliable detection of a LPPL regime confirming the existence of a stock bubble. Using the methodology presented above, we believe that the formation and bursting of the bubble could have been predicted several months before the crash. However, a very precise date of the crash seems harder to identify. Although we obtain ex ante estimates of the crash date close to the actual date, the estimates are not as robust as one would have hoped.

\subsection{Extrapolating the future behaviour of the LJSE index}

In this last subsection we use the methodology developed by Sornette and Johansen (Johansen et al. 1999a) and briefly presented in Section 2 to analyse the potential future behaviour of the LJSE index. More precisely, we employ the methodology developed for fitting antibubbles to try to predict the future behaviour of the LJSE index. In order to do this, we fit equations (2) and (3) on the same data set as before. In the case of antibubbles we set $t_{c}$ which denotes the change of the stock regime from a bubble to an antibubble phase equal to 27 September $2007^{3}$. The last date of our data set is 21 May 2010. We thus have approximately three years of data for determining the dynamics of the antibubble phase.

The resulting figures (see Appendix Figures 7 and 8) show the overall fit is very good, whereas the values of the parameters denoting antibubble behaviour differ quite substantially when comparing equations 2 and 3 . In the case of the simple model (equation 2) the values of the power exponent and angular frequency are close to the range of optimal values obtained for other countries (Johansen et al. 2000 a). The power exponent in this case is equal to 1.03 , whereas the angular frequency is equal to 5.9 , which is the value one is most likely to expect with antibubble-like behaviour. Compared to values for other countries the Slovenian antibubble phase corresponds most closely to the antibubble phase in the Netherlands, USA-Dow Jones, Germany, Norway, Switzerland and the United Kingdom with values of the power exponent close to or slightly greater than 1 (Zhou et al. 2003).

Looking at the value of the power exponent one can conclude that the overall behaviour from September 2007 to May 2010 indicates the steep downward overall acceleration of the index (Zhou et al. 2003). As indicted by Sornette and Zhou (Zhou et al. 2003), a steep downward acceleration is compensated by a large amplitude of log periodic oscillations. As the values for the second fit indicate, if one includes second order

3 This is the last time the index breaks the 12,100 point mark; between the peak date and 27 September the value of the index oscillates around the 12,000 mark, with no apparent trend, whereas after 27 September there is a clear downward trend. 


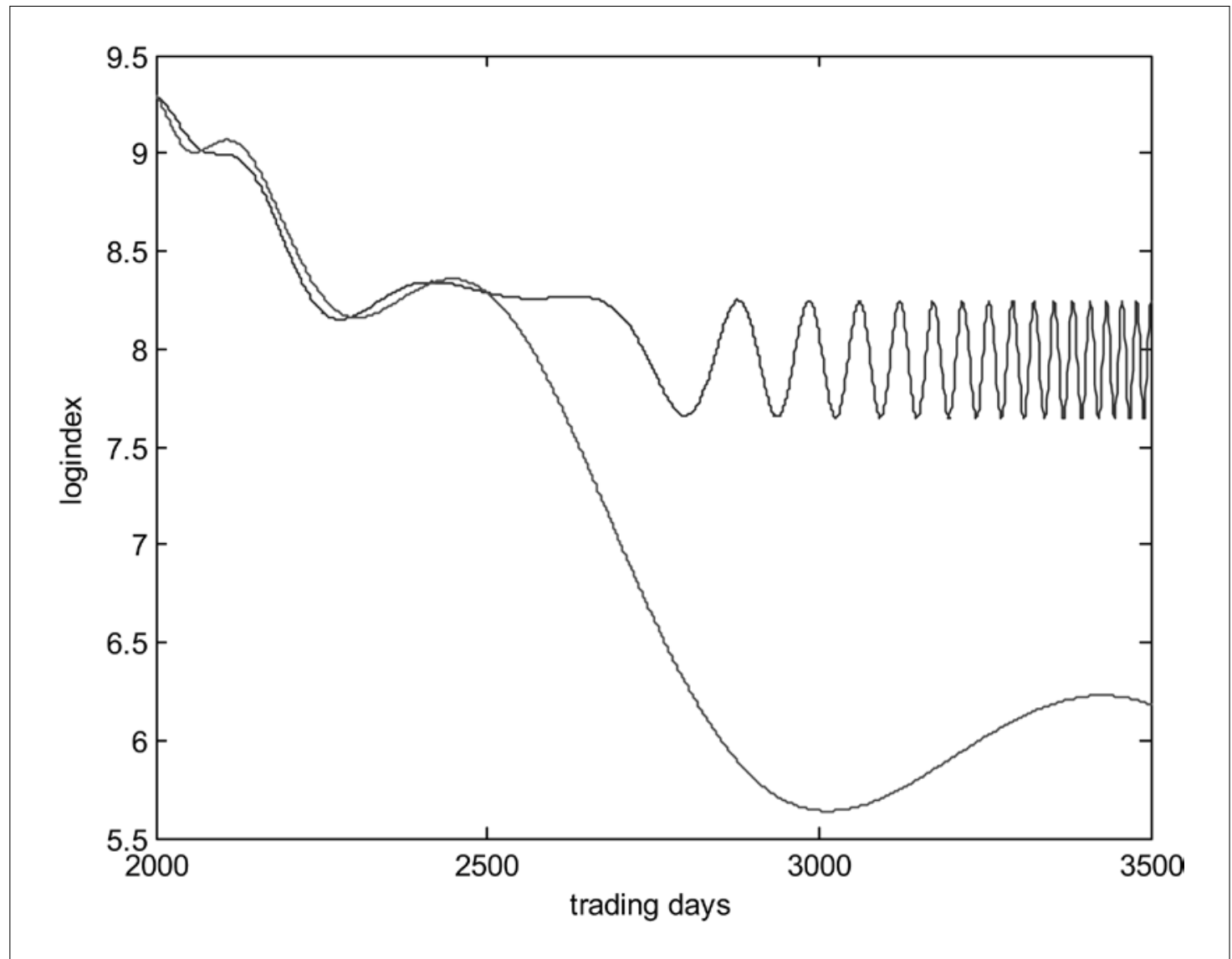

Figure 3: Predicting future behaviour of the LJSE index from equations 2 (upper oscilating line) and 3 (lower line) for the antibubble phase. The y axis gives the log (index), whereas the $x$ axis gives trading days measured from January $3^{\text {rd }}$ 2000. Source: LJSE and own work

harmonics the power exponent is indeed larger than 2.3, which shows that we are indeed facing a regime of steep downward acceleration, especially during the September 2007-September 2008 period. In contrast to equation 2 the values of parameters indicate that for values of $t>t_{c}$ there is a change of regime from downward sloping to oscillating with an amplitude of around 1,000 points. This can be largely confirmed by looking at the predictions of the future behaviour of the stock index using the results of fits of equations 2 and 3 up to May 2010 extrapolated until around mid-2015.

As one can see from the graph there are important differences between the two models. The simple model with no second order harmonics indicates a further steep decline of the index. However, this is not only the consequence of the data but a consequence of the model. With this model no stationary oscillatory behaviour is possible, the index can only be decreasing albeit at a slower or faster pace, but there cannot be a change in regime as is possible with equation 3; the extended model. The hypothesis that the first model might not be completely appropriate for describing the behaviour of the LJSEX in the period from September 2007 to May 2010 is confirmed by the values of the quality of fit. Namely, in the second case the $\mathrm{R}^{2}$ is some $40 \%$ smaller, although only two parameters are added to the model.
Due to the significant increase in the quality of fit when predicting the future behaviour of the LJSEX we put more emphasis on the extended model (equation 3). From the values of the parameters obtained by fitting equation 3 to the September 2007 to May 2010 period we can conclude that over the next few years we are most likely to experience a period of increased volatility with no clear increasing or decreasing growth pattern.

One should be careful when interpreting the results of future forecasts for the LJSEX using either of the models presented in Section 2. Namely, as already pointed out in Johansen et al. (1999c) stock indexes do not only behave according to either bubble or antibubble regime models since their movements can also be random with no apparent underlying regime. In-fact, this regime is most likely and thus we have to take into account that each antibubble phase may shift to some other regime such as a bubble regime or complete random behaviour. As indicated by Johansen et al. (1999c), the probability of such a regime shift is increasing in time and one cannot exclude the fact that one or several years in advance the antibubble phase model will prove inadequate for describing the dynamics of the LJSEX. If however such a shift in regime is not present, we may expect the increased volatility of the LJSEX with no apparent downward or upward trend for several years to come. 


\section{Discussion}

As noted in the previous section, the LJSEX clearly had a bubble-like pattern between 2005 and 2007. Notably, the values of the parameters were robust in terms of increasing time windows and dependence of the critical time with respect to shrinking time windows indicated that the herding behaviour was known for some time in advance and thus that the possible peak and consequent crash could have been predicted within a reasonable margin of error. This in itself is an important conclusion further indicating that the methodology proposed by Johansen and Sornette $(1998,1999$ a.b $)$ and extended by others is also successful in predicting bubbles in the case of the Slovenian stock market.

Although one can be content with the realisation that the methodology of bubbles gives precursory indications of a bubble forming, one is left to ask if there were other indicators or perhaps factors influencing/accompanying the formation of the bubble.

One possible answer here could perhaps be provided by studying the interdependence of interest rates, the amount of newly issued debt and the LJSE index. Namely, like in some other countries (most notably the USA and the UK) we are led to believe that low interest rates and lax lending standards establish grounds for the positive feedback herding behaviour that led to the bubble and the consequent crash. We believe that, similarly as in some other cases, initial positive economic indicators of Slovenian companies induced by strong global demand attracted investors which pushed up the prices, this created additional expectations in the general public and attracted less sophisticated investors. The additional leverag- ing available through the banking system together with low interest rates created surplus demand which caused prices to skyrocket and form a regime of self-fulfilling expectations. Finally, the bubble burst as some investors started cashing in their gains or new money/debt became more difficult to obtain.

In this section we try to test to what extent the bubble was coupled/influenced by the low interest rates and more flexible debt arrangements offered by banks. Second, we want to find out whether the crash preceded the drop in credit activity (especially asset-backed credit) or the other way around.

We look at interest rates for short-term consumer credit available to households (consumer credit) with variable adjusting interest rates or a fixed interest rate up to a period of one year. Further, we also look at the interest rate charged for short-term loans to non-financial institutions (it would have been preferable to obtain data on interest rates charged for asset- or stock-based loans, however such data are not available). This is because in Slovenia large investors were investing in the stock market via large financial holdings that had massive leverage, relatively little capital and a lot of debt, primarily short-term debt. Since short-term debt to financial holdings has the shortest duration of all types of debt, any worsening of credit conditions will be felt the most by holders of short-term debt. The fact that financial holdings were large players on the stock market can be seen by looking at the amount of short-term debt owed by the 10 largest financial holdings which, at the beginning of 2009, was well over EUR 1 billion (http://www.dnevnik.si/novice/aktualne_ zgodbe/1042352431). To give the reader a sense of relativity, daily turnover on the Ljubljana stock exchange amounts to

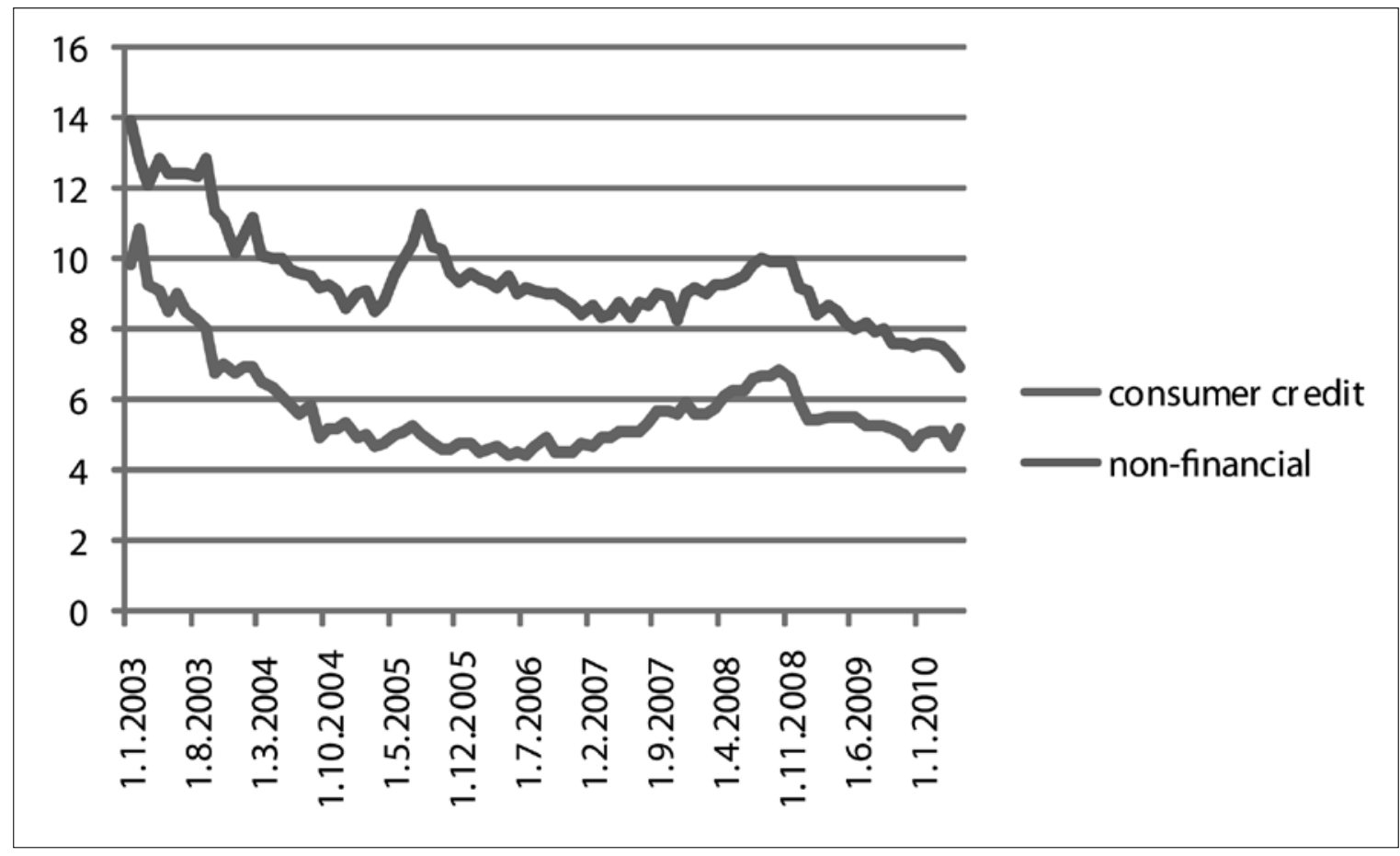

Figure 4: Interest rate for short-term consumer credit (lower line) and short-term credit to the non-financial sector (upper line); Source: Bank of Slovenia 


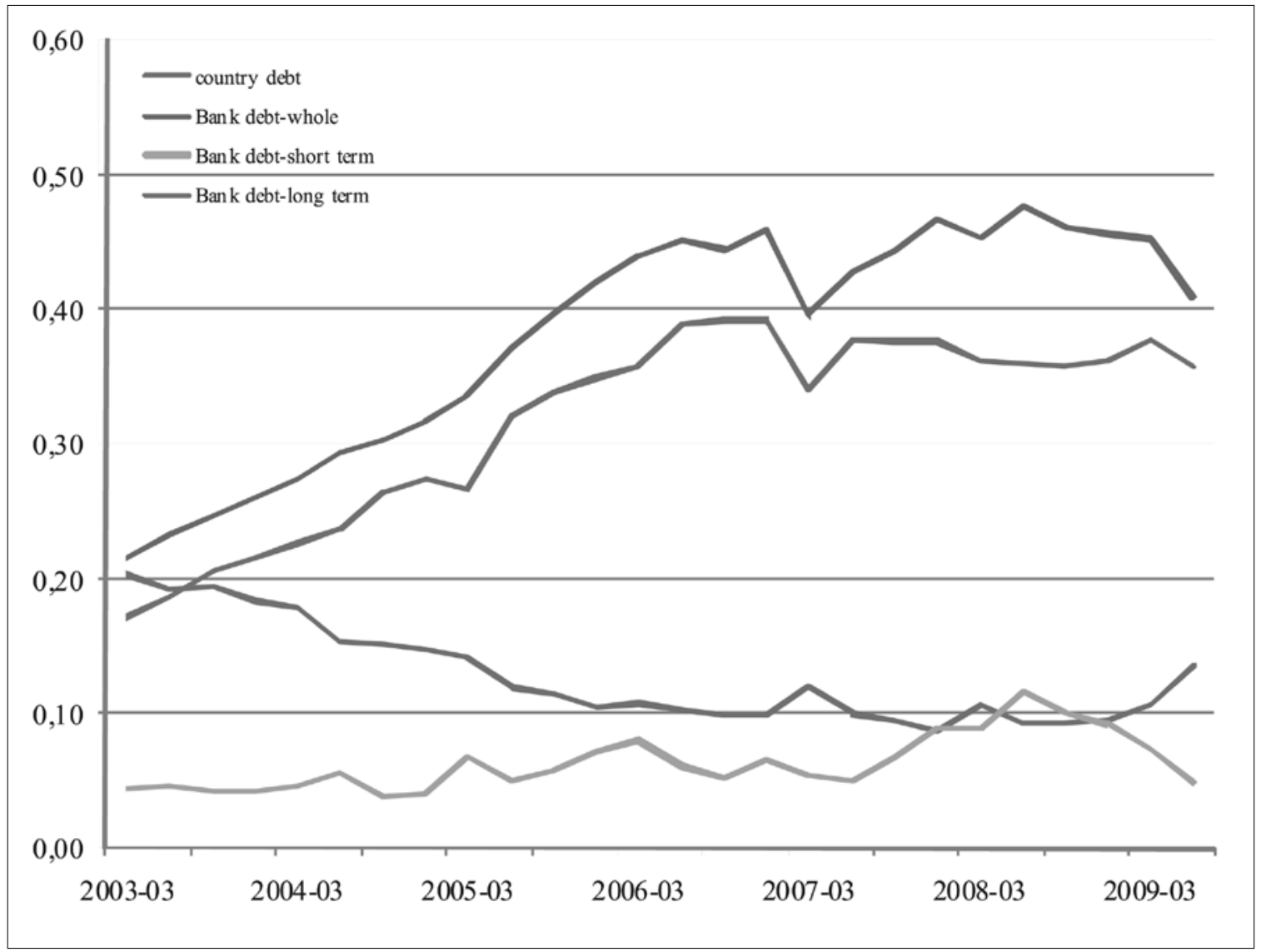

Figure 5: Share of state debt and bank debt as a percentage of whole debt (source: Simoneti et al (2010))

several million $€$ on average (http://www.ljse.si/cgi-bin/jve. cgi?doc=718\&sid=, Stock exchange).

As one can see the fall in interest rates coincides well with the growth in the LJSEX. In addition, observe that at the beginning of 2007 interest rates on both consumer credit and credit to the non-financial sector started rising which could indicate that the worsening of credit conditions acted as a trigger for the end of the stock market bubble. Another factor that probably contributed to the worsening of credit conditions was the increase of lending standards which manifested itself in stronger demand for collateral and an increased pick up (add on) of interest rate charges for asset-backed loans over the risk-free interest rate. Both factors are quite difficult to measure and thus this issue is left for further research.

Regarding the second question, we present the figures for credit activity measured by new outstanding debt to the nonfinancial sector and compare their dynamics to the dynamics of the stock market.

Observe that the growth of credit coincides well with the beginning of the bubble. The banks' credit activity (measured as both newly issued debt and short-term debt) picked up significantly in 2005, 2006 and 2007. If there is a clear correlation between the early phase of the bubble and the increased credit activity of the banking sector, the link between the end of the bubble and the restriction of credit activity is less pronounced.
As mentioned, the stock market peaked on 31 August 2007, while credit activity continued for some time after that date. Thus one is left to believe that deflation of the bubble was not linked/influenced by the contraction of bank lending. This conclusion may however be somewhat premature since we can observe that in the second quarter of 2008 there was already a contraction of long- and short-term lending which coincides with the period of the fastest decrease in the value of the stock index. In the last three quarters the LJSE index fell more than $60 \%$. In the previous three quarters the drop was just slightly above $25 \%$. One possible explanation of the stock market dynamics is that the initial contraction was spurred by a tightening of lending standards which manifested itself in stronger demand for collateral, whereas in the second phase a contraction of the availability of lending acted as a further catalyst for the drop in the stock index.

We should stress that the analysis presented in this section is based on publicly available data, which is not as detailed as one would have hoped to draw a more powerful conclusion regarding the factors that caused the bubble to inflate and the subsequent drop in the stock index. In order to obtain more conclusive evidence one would need more detailed data on foreign investments in the stock market, data on the tightening of lending standards and data on the debt outstanding to financial holdings. The interplay between these factors and 


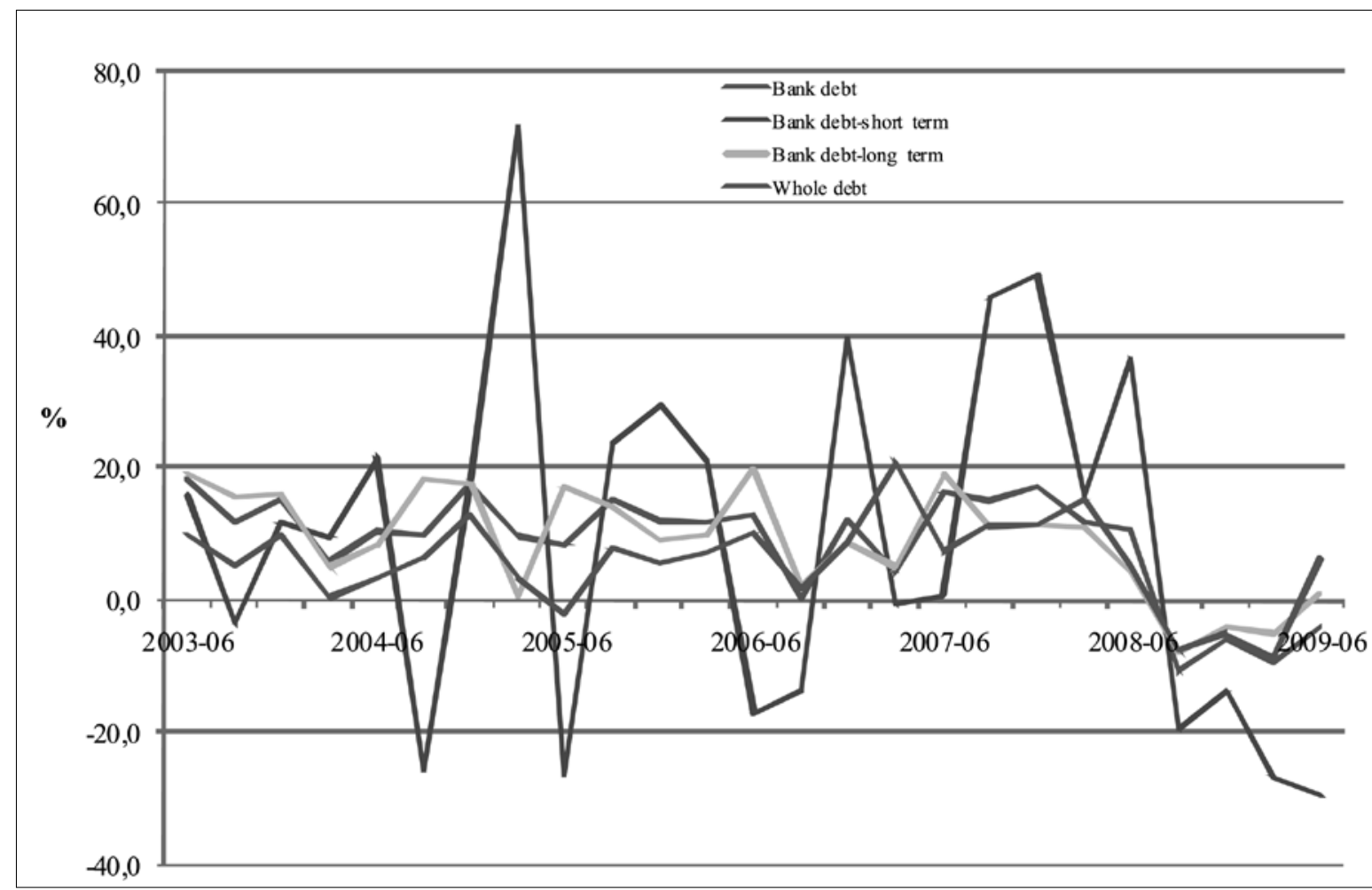

Figure 6: Growth rates for bank short-term debt, long-term debt, whole debt (source: Simoneti et al (2010))

the testing of the aforementioned hypothesis of the bursting of the Slovenian stock market bubble is left for further research.

\section{Conclusion}

In this paper we tested the sustainability of growth of the LJSE index in the 2000 to 2007 period and thereafter used the methodology developed by Sornette and Johansen (1998,1999a,b). The methodology is employed using data from the LJSE index from 3 January 2000 to 5 July 2010.

In the first part of the paper we tested whether a bubble was present in the period from 2000 to 2007 . By using the method of increasing time windows we found that the bubble most likely started around August 2005. As we now know (ex post) the bubble culminated on 31 August 2007. By using the method of increasing time windows we concluded that the bubble could have been predicted up to a few months in advance. On the other hand, a very precise date of the crash seems harder to identify. Although we obtained ex ante estimates of the crash date close to the actual date, the estimates were not as robust as one would have hoped. In Section 5 we sought to test the extent to which the bubble was coupled/ influenced by the low interest rates and more flexible debt arrangements offered by banks. We found that the growth of credit coincides well with the beginning of the bubble. Banks' credit activity (measured as both newly issued debt and shortterm debt) picked up significantly in 2005, 2006 and 2007. If there is a clear correlation between the early phase of the bubble and the increased credit activity of the banking sector, the link between the end of the bubble and the restriction of credit activity is less pronounced. In the last section we tried to predict the future behaviour of the LJSEX using the methodology for antibubbles (Johansen et al. 1999a, $2000_{\mathrm{a}}$ ). We found that the extended antibubble model (equation 3) gives a good description of the market dynamics from September 2007 until May 2010. From the values of the parameters obtained in this way we conclude that in the next few years we are likely to experience a period of increased volatility with no clear increasing or decreasing growth pattern.

\section{Literature}

Blanchard, O.J. (1979). Speculative bubbles, crashes and rational expectations, Economic Letters, 3(4): 387-389, DOI:10.1016/ 0165-1765(79)90017-X.

Caprirolo, G., Lavrač, V. (2003). Monetary and exchange rate policy in Slovenia, Ezoneplus Working Paper No. 17G.

Cvijovic, D., Klinowski, J. (1995). Taboo search: An approach to the multiple minima problem, Science, 267 (5198): 664-666, DOI: $10.1126 /$ science. 267.5198 .664

Diba, B.T., Grossman, H.L. (1988). Explosive rational bubbles in Stock Prices?, American Economic Review, 78(3): 520-530, DOI: $10.2307 / 1809149$

Feigenbaum, J. A. (2001). A statistical analysis of log-periodic precursors to financial crashes, Quantitative Finance, 1(3): 346 360, DOI: 10.1088/1469-7688/1/3/306.

Feigenbaum, J. A. (2001). More on a statistical analysis of logperiodic precursors to financial crashes, Quantitative Finance, 1(5): 527 - 532, DOI: 10.1080/713665875. 
Froot, K.A., Obstfeld, M. (1991). Intrinsic bubbles: The case of Stock Prices, American Economic Review, 81(5): 1189-1214, DOI:10.1257/aer.89.5.1372.

Johansen, A. and Sornette, D. (1998). Stock market crashes are outliers, European Physical Journal B, 1(2): 141-143. DOI : $10.1007 / \mathrm{s} 100510050163$.

Johansen, A. and Sornette D. (1999a). Financial anti-bubbles: Logperiodicity in Gold and Nikkei collapses, Int. J. Mod. Phys. C, 10(4): 563-575, DOI:10.1142/S0129183199000437.

Johansen, A. and Sornette D. (1999b). Critical Crashes, RISK, 12(1): 91-94.

Johansen, A., Sornette D. and Ledoit, O. $\left(1999_{\mathrm{c}}\right)$. Predicting Financial Crashes using discrete scale invariance, Journal of Risk, 1(4): 5-32, DOI=10.1.1.50.3129.

Johansen, A. and Sornette D., $\left(2000_{\mathrm{a}}\right)$. Bubbles and anti-bubbles in Latin-American, Asian and Western Stock markets: An empirical study, Int. J. Theor. Appl. Fin., 4(6): 853-920, DOI: 10.1142/ S0219024901001218.

Johansen, A. and Sornette D. $\left(2000_{\mathrm{b}}\right)$. Evaluation of the quantitative prediction of a trend reversal on the Japanese stock market in 1999, Int. J. Mod. Phys. C, 11(2): 359-364, DOI: 10.1.1.35.3396.

Johansen, A. and Sornette D. $\left(2000_{\mathrm{c}}\right)$. The Nasdaq crash of April 2000: Yet another example of log-periodicity in a speculative bubble ending in a crash, Eur. Phys J. B, 17(2): 319-328, DOI: 10.1.1.30.7938

Johansen, A. and Sornette D. (2002). Large Stock Market Price Drawdowns Are Outliers, Journal of Risk, 4(2): 69-110, DOI: 10.1.1.31.7872.

Simoneti, M., Abramovič, K., Damijan, J., Masten, I., Mastnak, S., Mrak, M., Rojec, M., Berk Skok, A., Šušteršič, J., Vesnaver,
L.,(2010), Razvojne priložnosti trga kapitala v Sloveniji po finančni krizi, IER. [Developing capital markets after the financial crisis in Slovenia]

Sornette, D., Johansen, A. (2001). Significance of log-periodic precursors to financial crashes, Quantitative Finance, 1(4): 452 471, DOI: 10.1.1.67.8436.

Sornette, D. (2003). Why Stock Markets Crash (Critical Events in Complex Financial Systems), Princeton University Press.

West, K.D. (1987). A specification test for speculative bubbles, Quarterly Journal of Economics, 102(3): 553-580, DOI: $10.2307 / 1884217$.

Zhou, W. X., Sornette, D. (2007). A case study of speculative financial bubbles in the South African stock market 2003 - 2006, Physica. A, 388 (6): 869-880, DOI: 10.1016/j.physa.2008.11.041.

Zhou, W. X., Sornette, D. (2006). Is there a real estate bubble in USA?, Physica A: Statistical Mechanics and its Applications, 361(1): 297-308, DOI: 10.1016/j.physa.2005.06.098.

Zhou, W. X., Sornette, D. (2003). Evidence of a worldwide stock market log-periodic anti-bubble since mid-2000, Physica A: Statistical Mechanics and its Applications, 330(3-4): 543-583, DOI: 10.1016/j.physa.2002.12.001.

Aleš Ahčan is an Assistant Professor in Actuarial Science in the Department of Finance and Banking of the Faculty of Economics at the University of Ljubljana, Slovenia. His current research projects focus on pricing derivatives in incomplete markets, Bayesian mortality forecasting, predicting stock bubbles and pension problematic. 


\section{Appendix}

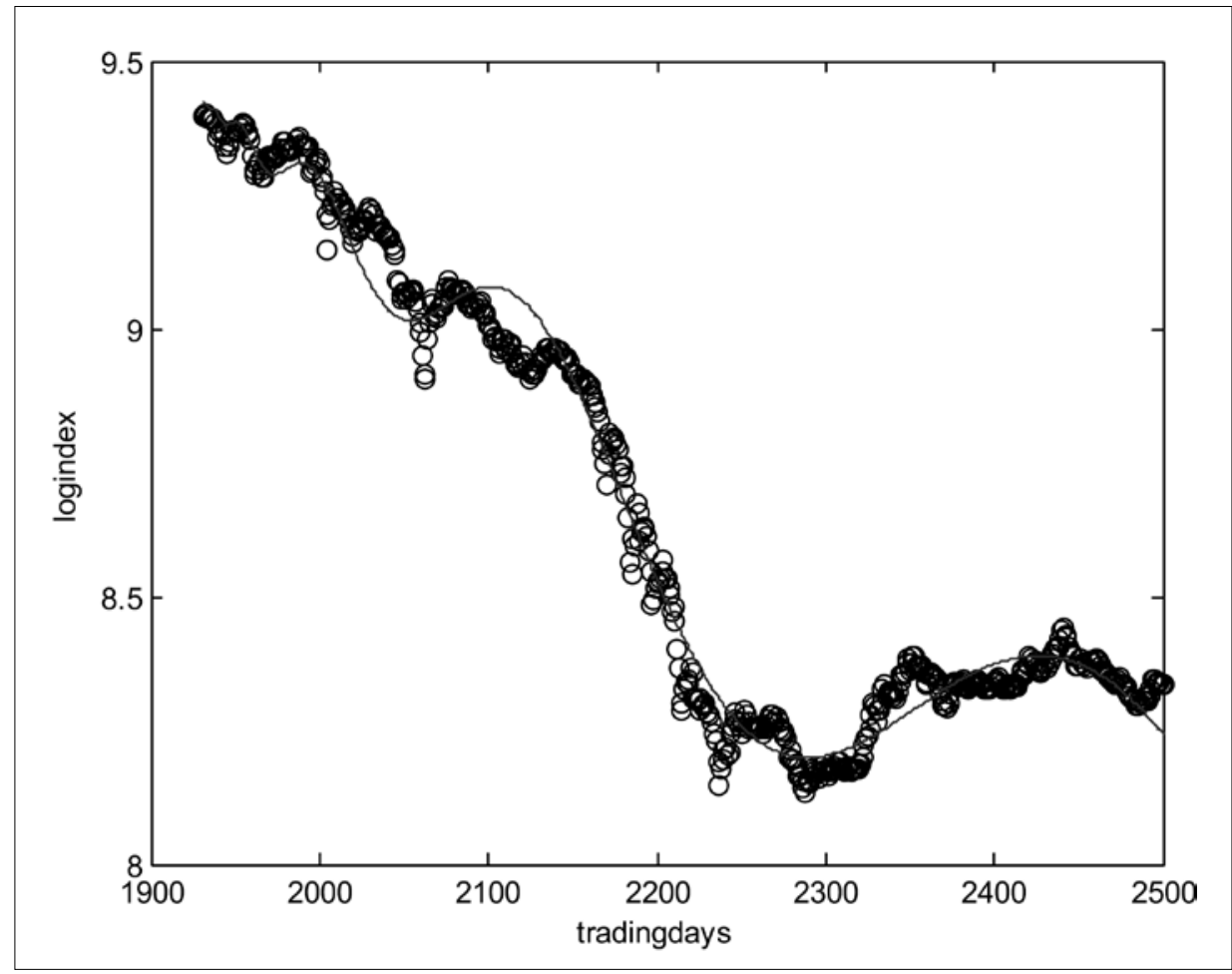

Figure 7: Fit of the antibubble phase using equation 2.. The y axis gives the log of the index value, whereas the $x$ axis gives the date evaluated as trading days from 1 January 2000 onwards. The values of the parameters for the fit are B=-0.0023 1.0324 $C=-0.69 * 10^{-3} \quad \omega=5.9148 \quad \phi=6.8271 \quad A=9.4248$ tc $=19.3000$

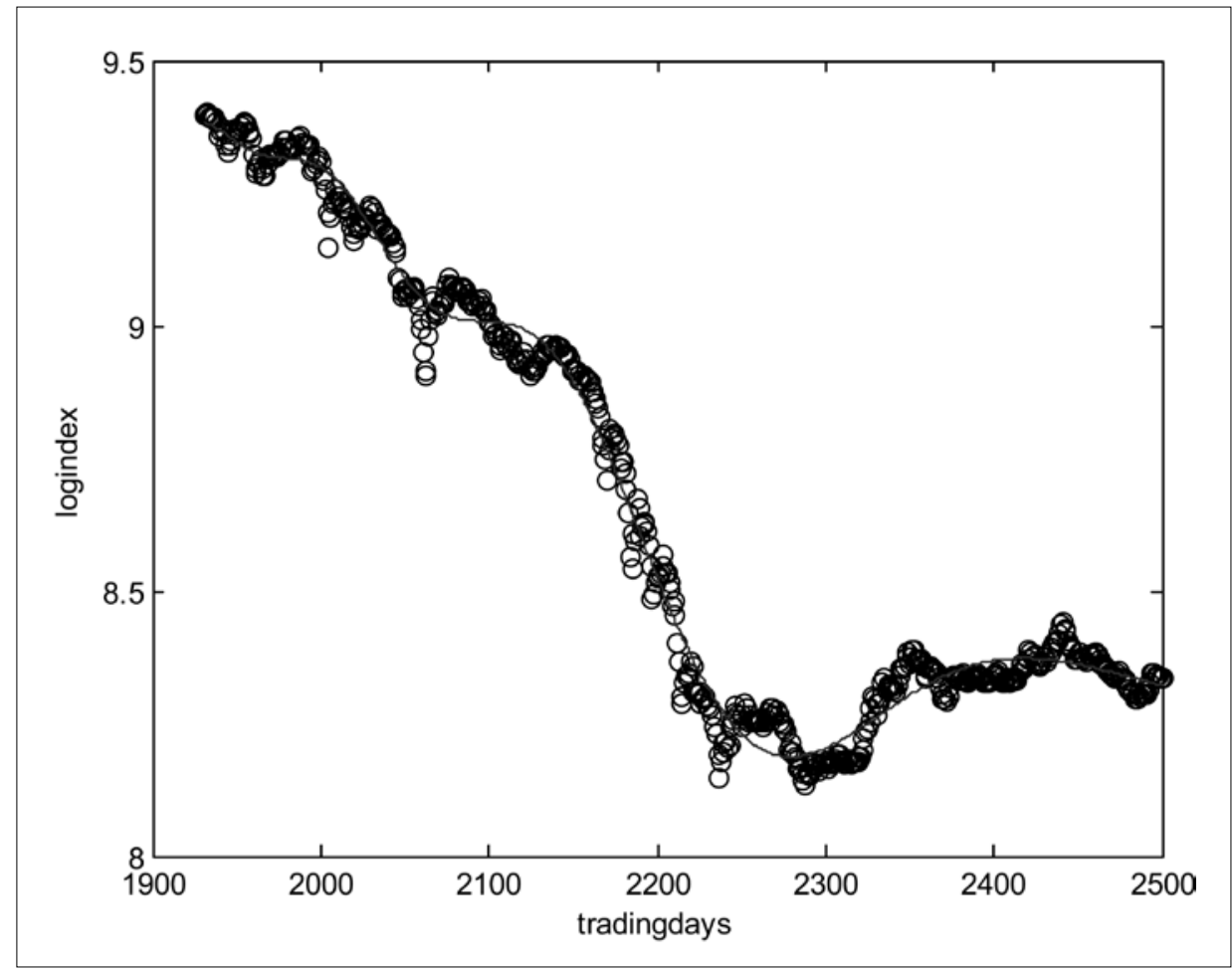

Figure 8: Antibubble fit using equation 3. The values of the fit $A=9.41, B=-14 * 10-6, m=2.3, C=28 * 10-7, \omega=9.75, \phi=-14.3, t c=1850$, $\Delta \omega=-0.83, \Delta t=1.1$. The $y$ axis gives the log of index value whereas the $x$ axis gives the date evaluated as trading days from 1 January 2000 onwards. Only the values of the index from September 2007 are plotted. 